\title{
Erziehungswissenschaftliche Lexika als Material zur Erforschung der Disziplingeschichte? Theoretisch-methodologische Überlegungen und inhaltliche Einblicke
}

\author{
Anne Hild und Anna Stisser \\ Georg-August-Universität Göttingen
}

\begin{abstract}
Eine etablierte erziehungswissenschaftliche Lexikonforschung gibt es noch nicht. Doch haben einige Studien in den letzten Jahren auf den besonderen Stellenwert von Lexika (vgl. z. B. Lenzen und Rost, 1999; Brachmann 2008; Tenorth, 2001; Herzog, 2005, Rost, 2008; Großkopf, 2012), Handwörterbüchern und Enzyklopädien im wissenschaftlichen Kommunikationszusammenhang hingewiesen. Demnach können (fach-)lexikalische Formate als Wissensspeicher beschrieben werden, die sie für verschiedene Analysen fruchtbar macht. Wir wollen hier der Frage nachgehen, inwiefern sich Lexika für die Erhellung disziplingeschichtlicher Fragestellungen eignen. ${ }^{1}$
\end{abstract}

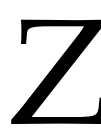
UNÄCHST werden wir die Eignung von Lexika, ihre Charakterisierung als Wissensspeicher und Indikatoren für disziplinäre Kommunikation beschreiben, um anschließend Einblicke in unsere laufenden Dissertationen zu geben. Dazu werden wir zunächst unser Korpus - deutschsprachige pädagogische und erziehungswissenschaftliche Le- xika aus dem Zeitraum von 1774 bis 1989 - vorstellen. Beispielhaft für die Differenzierung der Lexika nach ihren Merkmalen rekonstruieren wir die von den Herausgebern in Vorwörtern dargestellten wissenschaftstheoretischen Positionierungen in den 1970er Jahren. In einem weiteren Schritt bieten wir methodische Einblicke - auch in die Virtuelle For-

\footnotetext{
${ }^{1}$ Überarbeite Fassung des Vortrags zur Wissenschaftlichen Tagung am Lehrstuhl für historische Pädagogik der Pädagogisch-Psychologischen Fakultät der Eötvös-Loránd-Universität Budapest vom 16. bis 17. Mai zum Thema: „Modelle der Erziehungswissenschaft in Mitteleuropa in der zweiten Hälfte des 20. Jahrhunderts - Forschungsergebnisse, theoretisch-methodologische Annäherungen“"
} 
schungsumgebung für Erziehungswissenschaftliche Lexikonforschung, kurz VFU ELF, die unsere Projekte rahmt und uns als Analyse- und später auch als Präsentationstool dienen soll. Im fünften Schritt präsentieren wir Ergebnisse, die aus quantitativen wie qualitativen Analysen der in Lexika beschriebenen Personen stammen. Dazu wird die Gruppe der Frauen fokussiert, die eine marginale Stellung innehat, nichtsdestotrotz aber interessante Einsichten bietet. Daran schließt sich unser Fazit an.

\section{LEXIKA ALS WISSENSSPEICHER}

Fachnachschlagewerke bieten ihrem eigenem Anspruch nach einen repräsentativen, der Logik und internen Systematik des fachlichen Zusammenhangs folgenden Begriffsapparat. Sie umfassen in der Regel die Grundtatbestände des Feldes, beziehen sich auf legitimierte Strategien zur Untersuchung und Darstellung strukturell typischer, institutionell verstetigter Handlungspraktiken, und sie informieren schließlich über favorisierte Motive, Figuren, Topoi, Heuristiken und Semantiken einer Wissenschaft. Lexikalische Formate bilden das Fachspracheninventar eines Diskussionszusammenhanges mit einem hohen Anspruch an Vollständigkeit und Differenziertheit ab. Demgegenüber stehen die in der Wissenschaftsforschung vielfältig analysierten Periodika (z.B. Keiner, 1999; Brachmann, 2008; Tenorth, 1986; Stroß und Thiel, 1998), deren Beiträge eher dazu die- nen, neue, unter Umständen noch vage Erkenntnisse schnell der wissenschaftlichen Forschungsgemeinschaft zugänglich zu machen (vgl. etwa Brachmann, 2008. S. 331.; Keiner, 1999. S.17.).

Daher kann ihre Analyse Aussagen über wissenschaftliche Interaktionen zwischen forschenden Gruppen und ihren Kommunikationsstrukturen ermöglichen. Auf der inhaltlichen Ebene sind Aussagen über schnelllebige thematische Konjunkturen und Innovationen möglich. Dagegen zeigen Stichworte in Lexika einen zum Herausgabezeitpunkt möglichst $a b$ schließenden Wissensstand an. Diese "abschließende Modellierung" (Brachmann, 2008. S. 331.) kann als Indikator für einen Übergang von Ergebnissen aus ihrem in den schnelllebigeren Periodika abbildbaren Produktionszusammenhang in einen konsolidierten, konsensuell geteilten Zustand eingestuft werden (ebd., S. 331.).

Dieser Funktion der Repräsentation eines Konsens an Wissen kann eine zweite, zukunftsorientierende, diskursbestimmende, setzende Funktion, nämlich die der Kanonisierung von Erkenntnissen, beigeordnet werden. Fachlexika repräsentieren Inhalte, die eine Aura des Gewichtigen umgibt - Herzog spricht vom „mainstream" der Disziplin. Von daher seien Lexika sehr geeignet, die „kognitive Gestalt" der Disziplin (Herzog, 2005. S. 676.) zu untersuchen. In Lexika wird dasjenige Wissen versammelt, das als wahr, anerkannt und relevant gilt.

Auch die verlegerischen Hürden bei einem solchen Prestigeobjekt sind 
hoch $^{2}$. Meist sind an der editorischen Bearbeitung eines Lexikons mehrere Personen beteiligt - und nimmt man die Autoren hinzu -, kommt man sogar auf mehrere hundert Personen, die sich über Jahre hinweg damit beschäftigen. Trotz des Anspruches Gesamtheit abzubilden, wird schon bei der Auswahl der Themen und der Autoren zwangsläufig selektiert. Häufig mit ideologischem, konfessionellem und/oder wissenschaftsprogrammatischem Hintergrund. Dieser Hintergrund ist dann oft durchgängig nachvollziehbar und macht die Untersuchung ertragreich.

Friedrich Rost (2008), macht darauf aufmerksam, dass insbesondere moderne Fachlexika meist als Auftragswerke entstehen und für Verlage mit hohem Kostenrisiko verbunden sind; in der Folge müssten seines Erachtens die Inhaltssetzungen weniger als Aussagen über die disziplinäre Formierung interpretiert werden, denn als Aussagen über ihre Marktkonformität. Außerdem würden sie vorwiegend für Laien, Lerner und die Profession konzipiert und nicht (unbedingt) für die Disziplin.

Auch diese Einwände liefern wertvolle Hinweise für die Eignung von Lexika als Analysegegenstand: Sie weisen nämlich darauf hin, dass die Lexika unbedingt in ihrem Entstehungszusammenhang betrachtet werden müssen. Ohne den Einbezug qualitativer Rahmendaten, also etwa Angaben zu den Zielgruppen, zu den durch Herausgeber oder Autoren vertretenen Schulen und wissenschafts- theoretischen Positionen oder weltanschaulicher Bindungen, ist die Analyse der in den Lexika repräsentierten Inhalte nur bedingt aussagekräftig.

\section{PÄdAgogische UND ERZIE- HUNGSWISSENSCHAFTLICHE FACHLEXIKA VON 1774 BIS 1989}

Insgesamt erscheinen uns Lexika in ihrer Eigenschaft als Wissensspeicher, deren serielle Analyse Aussagen über die epistemologische Dimension disziplinärer Entwicklung möglich macht, geeignet, um die Disziplingeschichte $\mathrm{zu}$ erforschen.

Auf der Grundlage von Lenzen und Rost (1999) und Brachmann (2008) haben wir für den uns gemeinsam interessierenden Zeitraum bis 198977 Auflagen von deutschsprachigen Nachschlagewerken identifiziert, die eine allgemeinpädagogische, das heißt wenigstens eine nicht-teildisziplinäre Orientierung, haben und nicht (allein) ratgebenden Charakter aufweisen.

In dem Dissertationsprojekt „Erziehung in Lexika" geht es um die Rekonstruktion dessen, was als Wissen der Disziplin zum zentralen Begriff der Erziehung in Fachlexika der Erziehungswissenschaft repräsentiert wird. Insbesondere werden Konjunkturen, (Dis-)Kontinuitäten, Aneignungen und Grenzmarkierungen dieses Wissens analysiert. Dabei werden die Veröffentlichungen zwischen 1895 und 1989 fokussiert. Ziel des Promotionsvorhabens ",Helden und Denker'“ ist es, alle Personen in pädagogischen

\footnotetext{
${ }^{2}$ Häufig kann man in Vorworten davon einen Eindruck bekommen.
} 
und erziehungswissenschaftlichen Lexika für den Zeitraum von 1774 bis $1945 \mathrm{zu}$ erfassen, die mit einem eigenen Stichwort vertreten sind.

Der Zeitraum beginnt also mit dem von Carl Daniel Küster herausgegebenen Band „Sittliches ErziehungsLexicon, oder Erfahrungen und geprüfte Anweisungen: wie Kinder von hohen und mittlern Stande, zu guten Gesinnungen und zu wohlanständigen Sitten können angeführet werden" aus dem Jahr 1774 (Küster, 1774). Es ist das erste Werk, das als pädagogisches Fachlexikon gelten kann (vgl. Brachmann 2008. S. 340.).

Am Ende des von uns untersuchten Zeitraums steht die mit zwei Bänden handlichere Ausgabe der großen „Enzyklopädie Erziehungswissenschaft" (Lenzen und Mollenhau- er, 1982-86), nämlich die „Pädagogischen Grundbegriffe", von Lenzen unter Mithilfe von Rost (1989). Nicht nur die politische Zäsur lässt uns unsere Erhebung zu diesem Zeitpunkt beenden: Ende der 1980er Jahre ist die nach realistischer und kritischer Wende expandierte Disziplin allgemein etabliert, sie erscheint zu diesem Zeitpunkt gut gesättigt, vielfältig und breit aufgestellt.

Wir haben nur Erstauflagen und solche Auflagen aufgenommen, die von seiten des Verlags als verändert und/oder überarbeitet markiert wurden. Bündelt man die Erscheinungsdaten der Lexika in Jahrzehnten und bezieht sich jeweils auf die letzte Ausgabe eines mehrbändigen Werks, ergibt sich folgende Verteilung (Abbildung 1$)^{3}$.

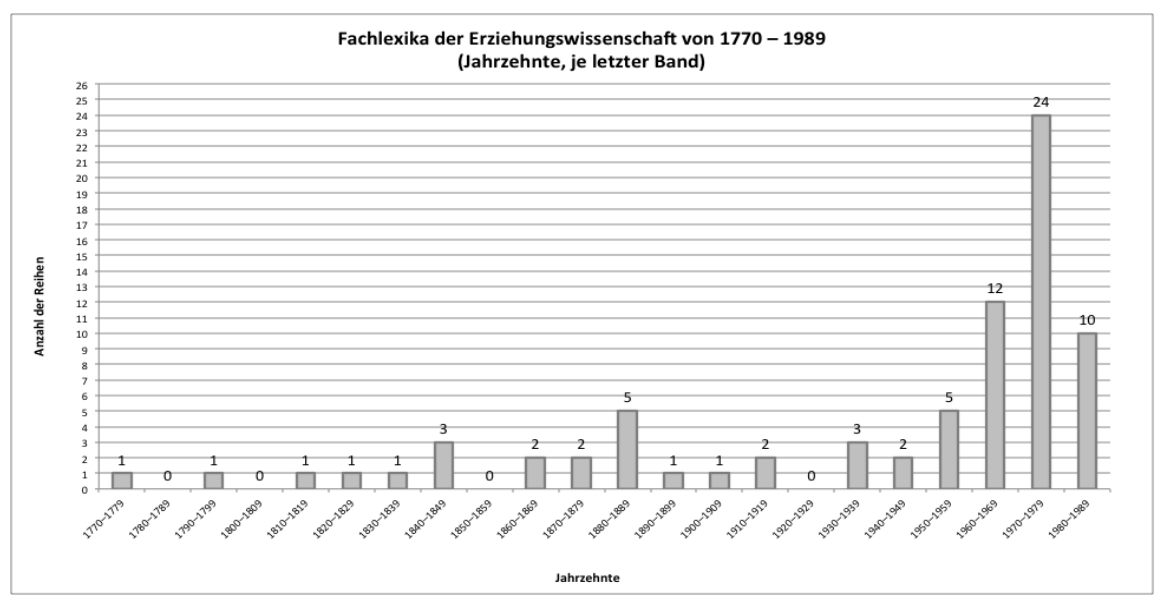

Abbildung 1: Verteilung der Lexika von 1774-1989

\footnotetext{
${ }^{3}$ Von den 77 Auflagen, werden insgesamt 51 vom jeweiligen Verlag als 1. Auflage bezeichnet. Ganz aussagekräftig ist dies allerdings nicht, denn darunter fallen auch Reihen, die von denselben Herausgebern und unter denselben Titeln firmieren, aber je als Erstauflage gekennzeichnet werden, obwohl etwa im Vorwort explizit auf die Vorgängerwerke Bezug genommen wird (etwa die Lexika von Roloff, Spieler, Rombach) als auch Lexika, die vollständig überarbeitet werden, mitunter sogar den Herausgeber wechseln, die sich aber als ",vollständig überarbeitete Auflage“ bezeichnen (Hehlmann, Böhm).
} 
Zunächst fällt die gleichmäßige Publikation von nur etwa einem Lexikon pro Jahrzehnt bis Ende der 1830er Jahre auf. In den 1840er erscheinen dann gleich zwei mehrbändige Auflagen von Münchs „Universal-Lexicon der Erziehungs- und UnterrichtsLehre" (Münch, 1840-42) sowie Hergangs zweibändige „Pädagogische Real-Encyklopädie“ (Hergang, 1843-47). Der nächste Gipfel wird in den 1880er Jahren erreicht; zwei vielbändige Lexika werden vervollständigt (Rolfus' Werk (1863-66), katholisch, und Schmids (1859-78), evangelisch).

Danach erscheinen im Mittel immer etwa ein bis zwei Werke pro Jahrzehnt bis zum Zweiten Weltkrieg. In den 1950er und 1960er Jahren steigen die Neuauflagen dann auf fünf und zwölf Auflagen. Für die 1970er Jahre verdoppelt sich diese Zahl auf 24 Nachschlagewerke. Diese besonders hohe Erscheinungsquote in den 1970er Jahren, kann nicht nur als Zeichen der Expansion der Erziehungswissenschaft gelesen werden - sondern auch als symptomatisch für eine in der epistemologischen Dimension feststellbare Diversifizierung innerhalb der nun flächendeckend institutionalisierten Erziehungswissenschaft. Dieser Peak provoziert also die Frage: Ist es schlicht mehr Wissen, das in mehr Lexika mündet - oder ist es vor allem diverseres Wissen, welches zu ganz unterschiedlich orientierten Lexika mit ganz unterschiedlichen Wis- sensbeständen führt?

\section{Positionierungen IN VORWORTEN VON FACHLEXIKA DER 1970ER JAHRE}

Berücksichtigt man, dass die hier untersuchten Lexika sich nicht primär an pädagogisch Handelnde richten, wird man annehmen, dass innerwissenschaftliche Gründe und Diskussionen um die sich wandelnde Erziehungswissenschaft - beispielsweise die zunehmende Orientierung am sozialwissenschaftlichempirischen Paradigma oder die aufstrebende kritische Erziehungswissenschaft - eine besondere Rolle spielen. Überprüfen lässt sich das (zumindest der Tendenz nach) durch die Lektüre der Vorworte, Einleitungen, Geleitworte und Klappentexte. ${ }^{4}$

Für die Motivation, ein Lexikon herauszugeben, lassen sich dort vereinfacht zwei Argumentationslinien identifizieren, die beide als zentrales Moment den aktuellen Wandel der Erziehungswissenschaft betonen. Im ersten Fall werden die sich wandelnden Erziehungs- und Bildungspraxen herangezogen, um den wissenschaftlichen Wandel zu begründen. Je nach wissenschaftstheoretischer Grundlegung hat Erziehungswissenschaft hier die Aufgabe, diese Praxis zu begründen, zu beobachten oder zu reformieren.

Die zweite Argumentationslinie

\footnotetext{
${ }^{4}$ Dass die Gattung des Vorworts insbesondere geeignet ist, um programmatische Muster herauszuarbeiten, konnte für verschiedene Formate gezeigt werden: Vgl. z. B. für Geschichten der Pädagogik Bühler 2012, für Einführungswerke in die Pädagogik Bühler 2014, für Deutsch-Lesebücher Selbmann 2006, für Schulgeschichtsbücher Jacobmeyer 2011.
} 
folgt dem Muster etwas verschoben: Es ist hier nicht primär die Praxis, die sich verändert, sondern die Wissenschaft selbst, weil sie sich von ihrer geisteswissenschaftlichen Tradition löst, sich Nachbarwissenschaften gegenüber öffnet und sich gesellschaftspolitisch positioniert. Damit öffnet sie sich auch gegenüber neuen Gegenständen und Methoden und erweitert so ihr Spektrum. In beiden Fällen wird die Neuherausgabe durch einen erweiterten erziehungswissenschaftlichen Wissensbestand gerechtfertigt. Daran schließt sich die Folgefrage an, inwiefern die Diversität des Wissens eine Rolle spielt.

Im Folgenden wollen wir dieser Frage anhand der von den Herausgebern ${ }^{5}$ angegebenen Selbstpositionierungen im wissenschaftstheoretischen Diskurs der 1970er Jahre nachgehen. Dem liegt die Annahme zugrunde, dass sich die Lexika so - über ihre bereits im Titel enthaltenen Setzungen und ihre je unterschiedlichen strukturellen Merkmale hinaus - in Cluster unterscheiden lassen.

Die Herausgeber selbst nehmen in immerhin zwölf der 24 Lexika $^{6}$ in den Vorworten Stellung zu Fragen der wissenschaftlichen Grundhaltung. Allerdings - und anders, als man erwarten könnte - ordnet sich ein Großteil der Lexika in den 1970er Jahren überhaupt keiner Richtung explizit $\mathrm{zu}$ - sondern grenzt sich höchstens von bestimmten Auslegungen ab. Ein Beispiel dafür sind erziehungswissenschaftliche Lexika in konfessioneller Tradition.

In den Jahren 1952 bis 1955 bringt das katholische Deutsche Institut für wissenschaftliche Pädagogik (DIwP) in Münster in Verbindung mit dem Institut für Vergleichende Erziehungswissenschaft in Salzburg (IVE) ein mehrbändiges „Lexikon der Pädagogik" heraus. Im Geleitwort betonen die Institute, dass der „Unordnung im Erziehungs- und Bildungswesen" (DIwP/IVE 1952. S. V) nur „vom Boden gesicherter und das Werk einheitlich tragender Grundanschauungen" (ebd.) begegnet werden könne. Diesen Boden biete „eine[...] bereits kraftvoll und vielseitig entwickelte[...] Erziehungswissenschaft, die ihre philosophisch begründeten Wahrheitsgehalte im christlichen Denken gesichert weiß"black(ebd.). 1964 erscheint ein Ergänzungsband; auch er bekennt sich zu einer „weltanschaulich geschlossene[n] Orientierung" (Schneider und Haase, 1964. S. V).

Im selben Jahr, 1964, formulieren die Herausgeber des katholischen Ergänzungsbands ihre Überzeugung, dass es „einen Grundbestand zeitlos gültiger Erziehungsaufgaben" (Schneider und Haase, 1964. S. V) gebe. Die ",christliche Erziehungswissenschaft"

\footnotetext{
${ }^{5}$ Es handelt sich im gesamten betrachteten Zeitraum durchgehend um Herausgeber und nicht um Herausgeberinnen.

6 Lexika in konfessioneller Tradition: 4 (DIwP/Speck/Wehle 1970, Rombach 1970/1971, Groothoff/Stallmann 1971, Rombach 1977); Lexika zur Kritischen Erziehungswissenschaft: 3 (Rauch/Anzinger 1973, Rauch/Anzinger 1975, Speichert 1975); sonstige Lexika: 5 (Horney u. a. 1970; Weber u. a. 1974, Wulf 1974, Roth 1976, Hierdeis 1978). Im Zeitraum davor werden nur vereinzelt Angaben zur wissenschaftstheoretischen Positionierung unternommen.
} 
(ebd.) soll diese beschreiben. Groothoff und Stallmann gehen schon einen Schritt weiter; hier hat sich erziehungswissenschaftliche Arbeit von der normsetzenden, konfessionell gebundenen Aufgabe entfernt. Christliche Normen sind aber auch bei ihnen grundlegend für "pädagogisches Denken"; sie postulieren: „Glauben und Erziehung haben etwas miteinander zu tun: Wenn es darum geht, die erzieherische Wirklichkeit zu begreifen und der erzieherischen Verantwortung gerecht zu werden, dann tun Pädagogik und Theologie gut, aufeinander zu hören" (Groothoff und Stallmann, 1961. o. S.; ebenso 1964).

Auch von evangelischer Seite ist in den 1960er Jahren von einer engen Bindung an das Christentum die Rede. Dem "Pädagogischen Lexikon" von Groothoff und Stallmann 1961 (und der nur wenig überarbeiteten 2. Auflage 1964) ist ein Geleitwort des Präsidenten des Deutschen Evangelischen Kirchentags beigegeben. Hier heißt es, das Lexikon bemühe sich „mit anerkennenswerter Ernsthaftigkeit, über den derzeitigen Stand der Pädagogik [...] umfassend, objektiv und in evangelischer Sicht zu unterrichten" (Thadden-Trieglaff, 1961).

Wissenschaftlichkeit wird auch hier mit der konfessionellen Bindung in Einklang gebracht. Interessanterweise widersprechen Groothoff und Stallmann in beiden Auflagen dem Geleitwort, indem sie unterstreichen, dass sie sich "nicht in der Lage sehen, aus unserem evangelischen Bekenntnis allgemeingültige Antworten auf pädagogische Fragen herzuleiten. Wir meinen, daß der Versuch einer ,Evangelischen Pädagogik' der Vergangenheit angehört" (Groothoff und Stallmann, 1961. o. S.; ebenso 1964).

Katholische und evangelische Lexika in den 1960er Jahren sind - trotz der Differenzen bei der Zuschreibung der Aufgaben einer christlichen Erziehungswissenschaft - wenigstens insofern noch eng an ihre Konfessionen gebunden, als pädagogisches Handeln und Denken jeweils nur als christlich gedacht werden können. In den 1970er Jahren ändert sich das.

So schließt Rombach 1970 eine "weltanschaulich geschlossene" Orientierung, wie sie noch für den Ergänzungsband von 1964 des „Lexikons der Pädagogik" propagiert wird, kategorisch aus: Das Lexikon "gehört nicht selber einer ,Richtung' an; jedoch mutet es sich wohlerwogene Urteile über strittige Fragen $\mathrm{zu}^{\prime \prime}$ (Willmann-Institut, 1970. S. V).

In der Neuauflage von Groothoff und Stallmann von 1971 geben die Herausgeber an, es sei „ein immer deutlicher sich abzeichnendes neues Selbstverständnis pädagogischer Wissenschaft im ganzen" (Groothoff und Stallmann, 1971. o. S.), zu erkennen; dies äußere sich vor allem darin, dass die „im Vorwort zur ersten Auflage [...] betonte Verpflichtung gegenüber der christlichen Tradition neu zu bestimmen" (ebd.) sei. Lemmata mit evangelischem Inhalt würden nun durch solche ersetzt, in denen das Verhältnis von Theologie und Pädagogik allgemein erläutert werde (ebd.).

Die Lexika in konfessioneller Tradition grenzen sich also angesichts 
der sich wandelnden Erziehungswissenschaft von ihren eigenen konfessionellen Bindungen $\mathrm{ab}$. Sie schreiben sich aber nicht definitiv einer anderen Richtung - etwa einer empirischen oder kritischen - zu, sondern beschränken sich auf die Markierung des Wandels oder wehren durch Abgrenzung mögliche Klassifizierungen $\mathrm{ab}$.

So heißt es im „Handbuch pädagogischer Grundbegriffe“" von 1970 (Speck und Wehle, 1970), die Herausgeber wollten "die Kritik der Sozialwissenschaften und der modernen Wissenschaftstheorie als Herausforderung" (Speck und Wehle, 1970. S. VVI) verstehen. Vor allem aber wollen sie sich von einem ,urgeschichtlichen und naiven Aktualismus" (ebd., S. VI) abgrenzen. Es wird also Position bezogen, aber keine definitive Aussage zur wissenschaftstheoretischen Grundlegung des gesamten Lexikons unternommen.

Auch für die anderen erziehungswissenschaftlichen Lexika in den 1970er Jahren gilt: Nur wenige beziehen eine eindeutige Position. $\mathrm{Zu}$ diesen gehören Lexika, die einen dezidiert sozialwissenschaftlichen Anspruch haben und sich aktiv von einer geisteswissenschaftlichen Ausrichtung abgrenzen ${ }^{7}$. Würde man sich diese Einschreibungen genauer ansehen ergäben sich - so der durch grobe Sichtung gewonnene Eindruck aber hier stark differierende Vorstellungen davon, was das heißt. In ei- nigen Lexika wird nämlich damit der Anspruch verbunden, empirische und kritische oder empirische und normative oder verstehende und erklärende Ansätze zusammen zu denken. Erziehungswissenschaft kann innerhalb eines einzigen Vorworts, als „Erziehungswirklichkeit erforschende Erfahrungswissenschaft" (Keller und Novak, 1979. S. 7.), als "Geistes- und Sozialwissenschaft" (ebd.), als ,normative Wissenschaft" oder als „Handlungsforschung" (ebd.) beschrieben werden. Wie auch in den Lexika in konfessioneller Tradition werden entweder verschiedene, auch einander ausschließende Charakterisierungen unternommen, ohne sich einer dieser Vorstellungen anzuschließen, oder aber vor allem Abgrenzungen betont.

Diesem Muster widersprechen schließlich nur die insgesamt zwei Lexika mit drei Auflagen, die ihren Gegenstand in dezidiert kritischer Perspektive betrachten und schon im Titel ihre Position Das „Kritische Lexikon der Erziehungswissenschaft und Bildungspolitik“, herausgegeben von der Zeitschrift päd. extra unter Leitung von Horst Speichert (1975) und die beiden Auflagen des „Wörterbuchs Kritische Erziehung" von Rauch und Anzinger aus den Jahren 1972 und 1975.

Ansonsten dominiert die Angabe, das Lexikon selbst nehme keine definitive Position im Streit um die wissenschaftstheoretische Grundlegung ein. Dabei wird aber häufig betont, dass

\footnotetext{
7 Diesen Bezug findet man etwa in den Vorworten von Groothoff und Reimers 1973, Horney u. a. 1970, Wulf 1974 und Weber und Domke 1974 - bei letzeren sogar im Titel: „Kleines sozialwissenschaftliches Wörterbuch für Pädagogen".
} 
den Autorinnen und Autoren freigestellt sei, Stellung auch zu solchen Fragen zu beziehen: „Im übrigen“, betont Rombach, ,trägt jeder Mitarbeiter die Verantwortung für seinen namentlich gekennzeichneten Beitrag selbst" (Rombach, 1977. o. S.) ${ }^{8}$.

Die Spannungen zwischen den Artikeln werden so zum besonderen Potential des jeweiligen Lexikons (sie seien, auch das wird mehrfach betont, ein Alleinstellungsmerkmal unter den vielen Neuerscheinungen). Freilich weisen die Herausgeber in diesem Zusammenhang nur selten explizit darauf hin, dass sie ja schon mit der Auswahl der Autorinnen und Autoren eine inhaltliche Schwerpunktsetzung vornehmen.

Diese Verantwortungsverschiebung befreit die Herausgeber möglicherweise davon, selbst eine eindeutige Stellung zu beziehen - hier mögen die Hinweise von Rost (2008), ein Lexikon sei eben immer auch ein kostenintensives und risikoreiches verlegerisches Großprojekt und müsse vor allem unter marktpolitischen Gesichtspunkten vertretbar sein, eine Begründung liefern.

Der hier auf die 1960er und 70er Jahren bezogene Einblick in den Versuch, die Lexika in ihren Rahmendaten, ihren Intentionen und Positionierungen in unterscheidbare Gruppen einzuteilen, zeigt, dass weniger trennscharfe denn feingliedrige $\mathrm{Ab}$ grenzungen der Lexika untereinander rekonstruiert werden können. Gruppierungen und Typen von Lexika kön- nen nur für je ein untersuchtes Moment gebildet werden. Und neben den hier nicht weiter dargestellten strukturellen Merkmalen - Zeiträume, Staaten, monographisches Format oder eine Vielzahl von Autoren, der Hintergrund der Herausgeber und die thematischen Schwerpunkte eines Werks, Angaben zur Auflage oder in Rezensionen - müssen Intentionen, Motivationen, Positionierungen aufgenommen werden. Aber auch die anvisierte Zielgruppe und Zuschreibungen über die Aufgaben der Erziehungswissenschaft sind relevant. Nur dann können auch die Wissensbestände in den Lexika sinnvoll eingeordnet werden.

\section{Methodologie}

Wir analysieren die Fachlexika inhaltlich auf mindestens drei unterschiedlichen Forschungsebenen.

Auf einer ersten Ebene werden die Lexika als Gesamtdatum betrachtet, das je für sich für eine bestimmte Zeit, eine bestimmte Schule oder Richtung steht. Hier bieten sich inhaltsanalytische Untersuchungen beispielsweise der Vorworte an. Darüberhinaus ist für diese Ebene der Entstehungskontext des Lexikons interessant.

Auf der zweiten Ebene spielen die Wissensbestände in den Lexika eine Rolle, die wir synchron und diachron zu anderen Einzelinhalten in Bezug setzen. Die Analyseeinheit ist hier das konkrete Lemma, das inhaltsanalytisch ausgewertet wird: Welche theore-

\footnotetext{
8 Ähnliche Aussagen finden sich auch z. B. bei Groothoff und Stallmann 1961, Ipfling 1974, Wulf 1974 und Roth 1976.
} 
tischen Bezüge sind erkennbar? Welche Personen werden überhaupt beschrieben? Wie werden sie beschrieben?

Auf der dritten Ebene steht der beschriebene Inhalt - beispielsweise die in verschiedenen Lexika beschriebenen Personen - unabhängig von ihrer lexikalischen Beschreibung. Die Person wird mit Kontextdaten angereichert, die aus der Literatur oder den Lemmata selbst stammen (z. B. ihre Tätigkeit, Geburts- und Sterbedaten, Konfession).

Auf der ersten Ebene kann man die Lexika gruppieren, Fälle kontrastieren oder vergleichen, und weiterführende Thesen im Anschluss formulieren. Auf der zweiten Ebene kann man die Wissensbestände in Bezug setzen zu den Gruppen, man kann Wissensbestände über Zeit-räume oder eben in Lexika mit ähnlichen oder stark voneinander abweichenden Merkmalen - vergleichen.

In der seriellen Analyse kann man schon an dieser Stelle herausfinden, welche Themen wann gesetzt werden, welche Personen wann als relevant markiert werden oder welche der interessierenden Begriffe vor welchem Hintergrund verschwinden oder (wieder) auftauchen. Auf der dritten Ebene lassen sich dann - wieder in Bezug zu den anderen Ebenen - auch quantifizierende Aussagen machen: Z. B.: Wann dominieren Personen, die der Reformpädagogik zugeschrieben werden können? Auf allen Untersuchungsebenen wird kontextuelles Wissen (also beispielsweise über Zuschreibungen von Schulenzugehörigkeiten etc.) mit einbezogen.

Zur Bewältigung eines solchen Datenaufkommens empfiehlt sich eine mächtige und flexible Datenbank (vgl. Gast und Leugers-Scherzberg, 2010). Daher bot es sich an, dass wir mit der Virtuellen Forschungsumgebung VFU ELF auf Grundlage von Semantic CorA arbeiten, die es als SemanticMediaWiki-Plattform ermöglicht, Relationen zwischen Entitäten semantisch zu beschreiben.

Mir ihr können formale Beschreibungen und Annotationen erstellt werden, worauf strukturierte, frei kombinierbare Abfragen auch nach Eigenschaften und/oder Metadaten durchgeführt und dynamische Sichten - wie beispielsweise Tabellen, Zeitleisten oder auch Landkarten - erzeugt werden können. Mit ihren technischen Funktionalitäten beantwortet die VFU methodologische Fragen danach, wie große Mengen digitaler Ressourcen verwendet werden können, die bereits weltweit zur Verfügung stehen, ohne die ebenfalls bereits vorhandenen relevanten Metadaten zu verlieren.

Die Software kann - gerade angesichts standardisierter Datenmassen an individuelle Anforderungen und

\footnotetext{
${ }^{9}$ Diese Plattform ist zur Zeit noch in Entwicklung. Es handelt sich um ein Kooperationsprojekt des Deutschen Instituts für Internationale Pädagogische Forschung in Frankfurt (DIPF), des Karlsruher Instituts für Technologie (KIT) und des Arbeitsbereichs Allgemeine und Historische Erziehungswissenschaft der Universität Göttingen mit dem Ziel, mit der Erweiterung Semantic CorA eine Software zur Verfügung zu stellen, mit der Virtuelle Forschungsumgebungen für die Historische Bildungsforschung realisiert werden können. Das Projekt wird von der DFG unter
} 
Eigenschaften der Daten angepasst werden ${ }^{9}$.

Zur qualitativen Auswertung bietet die VFU ein Kodierungswerkzeug, mit dem relevante Passagen markiert und mit Kodes oder beschreibenden Texten angereichert werden können. Diese Kodierung kann je nach gewählter Forschungsmethode in unterschiedlichen Verfahren vergeben werden.

Für explorierende und induktive Vorgehensweisen können die Kodes je neu und blind vergeben werden, d.h. man sieht die bereits vergebenen Kodes nicht.

Aber man kann auch mit deduktiven Verfahren bereits angelegte Kodes oder Kategoriensysteme vergeben und kann diese aus einer Vorschlagsliste auswählen. Im Anschluss kann man sich die Textstellen zu einem Kode gesammelt anzeigen lassen.

Dadurch, dass die VFU online zugänglich ist, können die erarbeiteten und erschlossenen Daten in einer Art wissenschaftlicher Wertschöpfungskette für SekundärforscherInnen erhalten bleiben. Außerdem können mehrere Personen gleichzeitig an demselben Datensatz arbeiten - und so möglichweise voneinander profitieren. Außerdem ist geplant, dass nach Abschluss der aktuellen Forschungs- arbeiten die VFU für ForscherInnen freigeben wird, so dass sie mit den erzeugten Daten weiter arbeiten können, möglicherweise auch nur im Sinne eines weiteren Nachschlagewerkes. $^{10}$

\section{Beispielanalysen ZU}

FRAUEN IN FACHLEXIKALISCHEN FORMATEN VON 1774 BIS 1942

Um alle in den pädagogischen und erziehungswissenschaftlichen Lexika vorkommenden Personen zu erfassen, wurden soweit vorhanden die Inhaltsverzeichnisse, Register oder die Lexika selbst durchgesehen und alle Personen identifiziert. Danach wurden die Personen bereinigt, beispielsweise Klarnamen und Pseudonyme abgeglichen und einer eindeutigen Person zugewiesen. Aktuell werden alle Personen mit Lebensdaten, Berufen u.a. angereichert.

In den 26 Lexika bis 1945 gibt es 15 Ausgaben, in denen insgesamt 72 Lemmata vorkommen, die Frauen beschreiben. In zwei Lexika des 18. Jahrhunderts des Gesamtkorpus' (nicht in Abb. 2) kommen noch gar keine Personenlemmata vor. Anfang des 19. Jahrhunderts erscheinen bereits vier Lexika mit zum Teil sehr zahlreichen Stich-

dem Titel „Förderung der Entwicklung einer Virtuellen Forschungsumgebung für die Historische Bildungsforschung mit Semantischer Wiki-Technologie - Semantic MediaWiki for Collaborative Corpora Analysis“ im Bereich Wissenschaftliche Literaturversorgungs- und Informationssysteme (LIS) gefördert.

Die Software wurde und wird auf unsere artikulierten Bedürfnisse angepasst, indem wir die für unsere Analyseabsichten relevanten Funktionalitäten beschreiben, die anschließend in der Plattform umgesetzt werden. Später werden die so entwickelten Erweiterungen für andere ForscherInnen zur Verfügung stehen. Die VFU ELF ist die erste auf Grundlage von Semantic CorA realisierte Forschungsumgebung. Siehe auch die Projektseite unter www.semantic-cora.org.

${ }^{10} \mathrm{Zu}$ näheren Erläuterung siehe Stisser/Hild/Ell/Schindler 2014. 
wortsammlungen ${ }^{11}$ und auch schon

Personenlemmata.

einigen relativ wenigen (22 und 51)
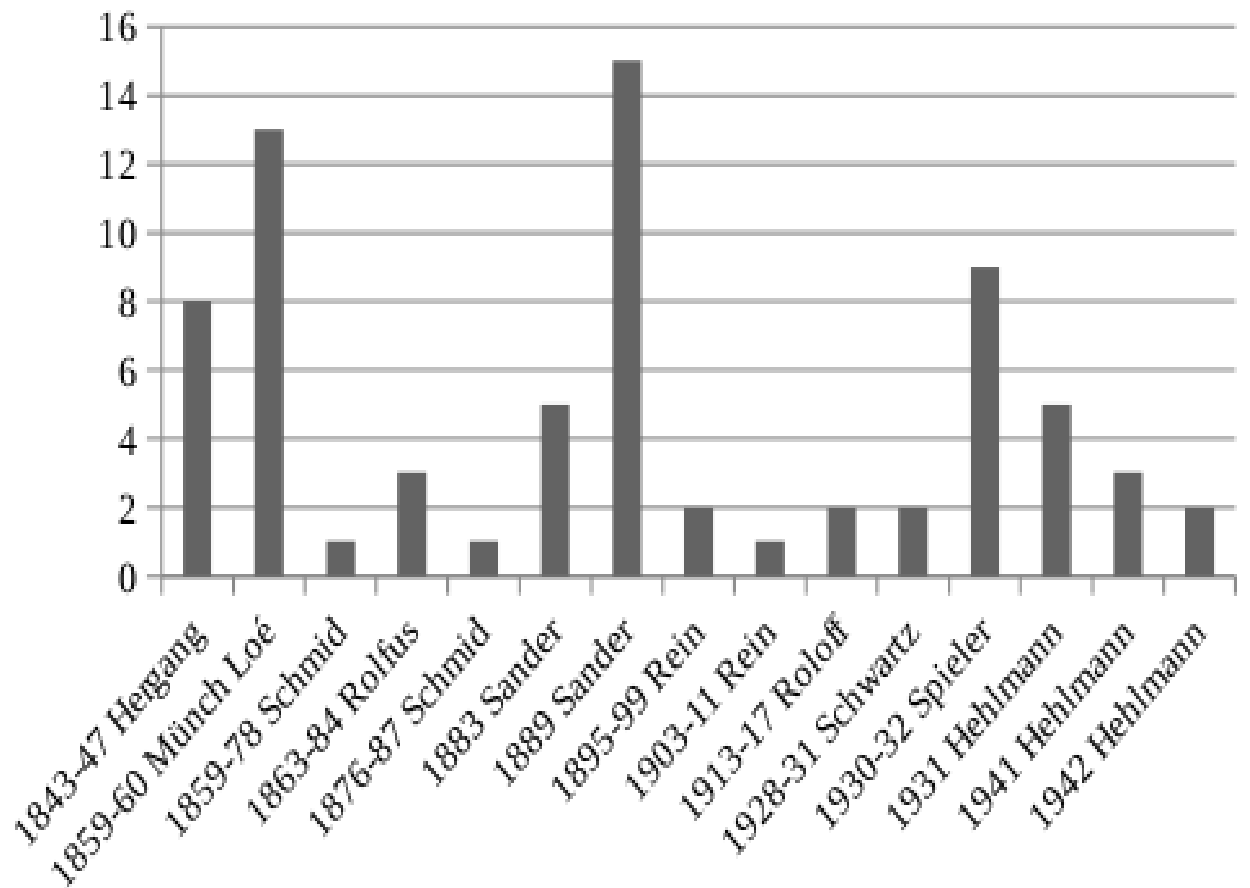

Abbildung 2: Menge der Lemmata, die Frauen beschreiben, für den Zeitraum bis 1945

Das erste einer Frau gewidmete Lemma erscheint dann im zweibändigen Lexikon von Karl Gottlob Hergang (1843). Es handelt sich zugleich aber - im Vergleich gesehen - um ein Lexikon mit zahlreichen, nämlich 8 Einträgen zu Frauen: Denn meist führen die Lexika nur sehr wenige Frauen beschreibende Lemmata (zwischen 2 und 4), nur in vier Lexika kommen mehr als 5 Frauenlemmata vor. Dies sind dann auch Lexika, in denen Personenlemmata durchweg einen großen Anteil an der Gesamtlemmatamenge bilden.

Auf die beschriebenen Personen bezogen heißt das: Von 1.212 Personen, die im untersuchten Lexikonkorpus mit eigenem Stichwort genannt werden, sind 39 Frauen, das entspricht einem Verhältnis von 3,2\% Frauen gegenüber 96,8\% Männer.Immerhin 18 dieser Frauen werden häufiger als einmal genannt:

\footnotetext{
${ }^{11}$ So hat das Lexikon von Baumgarten (1828) 508 Stichwörter, das von Wörle (1835) 322 Stichwörter und das Lexikon von Münch (1840-42) sogar 1.339 Stichworteinträge.
} 
Tabelle 1: Auflistung derjenigen Frauen, die mindestens zwei Stichworteinträge in verschiedenen Lexika aufweisen

\begin{tabular}{|c|c|c|c|}
\hline Lange, Helene & 5 & Gleim, Betty & 2 \\
\hline Lippe, Pauline Christine Wilhelmine zur & 4 & Ward, Mary & 2 \\
\hline Saussure de Necker, Albertine Adrienne & 4 & More, Hannah & 2 \\
\hline Marenholtz-Bülow, Bertha Maria von & 4 & Schallenfeld, Rosalie & 2 \\
\hline Key, Ellen & 4 & Wildermuth, Ottilie & 2 \\
\hline Montessori, Maria & 4 & Hrotsvita (Roswitha von Gandersheim) & 2 \\
\hline Rudolphi, Caroline Christiane Louise & 3 & Maintenon, Françoise D'Aubigny de & 2 \\
\hline Maria Theresia & 3 & Bühler, Charlotte & 2 \\
\hline Hamilton, Elizabeth & 2 & & \\
\hline
\end{tabular}

Die Liste wird in der Reihenfolge der Anzahl der Einträge angeführt von Helene Lange, die seit ihrer Erstnennung 1931 in Schwartz (Caspar, 1931) durchweg auch in den folgenden Lexika genannt wird. Auf dem zweiten Platz mit je vier Einträgen folgen: Fürstin Pauline zur Lippe, Albertine Saussure de Necker, Bertha von Marenholtz-Bülow, Ellen Key und Maria Montessori. Den dritten Platz mit je drei Nennungen teilen sich Caroline Rudolphi und Maria Theresia. Zweimal genannt werden neun Frau$\mathrm{en}^{12}$. Es handelt sich insgesamt um nur wenige Nennungen, so dass sich beispielsweise keine Verläufe feststellen lassen.

Das bis hierhin skizzierte Bild kann noch um Einzelergebnisse ergänzt werden. Es gibt zusätzlich zu den 72 Lemmata im Lexikon von Sander (2. Aufl. 1889) noch 5 Lemmata, die eine Frau in der Spitzmarke (also dem meist fettgedruckten oder ndersartig hervorgehobenen Teil) führen, aber keinen Erläuterungstext haben. Sie verweisen dafür auf die Stichworteinträge der Ehegatten bzw. geistigen Freunde der Frauen ${ }^{13}$.In diesen werden sie dann mit einem oder zwei Sätzen beschrieben. Das ist interessant, da beispielsweise eine der Frauen, Rosette Niederer-Kasthofer, als Schülerin und Kollegin Pestalozzis zu ihrer Zeit doch eine bekannte Frau war. Sie hatte die Leitung des 1806 von Pestalozzi gegründeten Töchterinsti-

\footnotetext{
${ }^{12}$ Mit nur je einer Nennung sind vertreten: Anhalt, Dorothea Maria von;Ausfeld, Regine Johanna; Campan, Jeanne-Louise-Henriette; Dransfeld, Hedwig; Frömmichen, Sophie; Genlis, Stéphanie Félicité de; Gnauck-Kühne, Elisabeth; Gumpert, Thekla von; Halberstadt, Wilhelmine; Hanke, Henriette Wilhelmine;Heinicke, Anna Katharina Elise; Hensel, Luise; Herber, Pauline; Hoffmann, Clementine; Lütkens, Dorothea Elisabeth;Paradies, Maria Theresia von; Scheppler, Louise; Schoppe, Amalie Emma; Stetten, Anna Barbara von; Weber, Helene; Zeller, Sophie.

${ }^{13}$ Es handelt sich um Christiane Dilthey, Rosette Niederer-Kasthofer, Jeanne-Françoise de Chantal, Jeanne-Marie von Gayette-Georgens. Das fünfte nurverweisende Lemma ist Louise Scheppler gewidmet, die aber auch anderer Stelle noch genannt wird, so dass sie in die Reihe der wenigstens zweimal genannten Frauen aufrückte.
} 
tutes ab 1809 inne, das Pestalozzi ihr 1813 schenkte, so dass sie ab dann formal alleinige Inhaberin war (vgl. Leimgruber, 2006, S. 54ff., 64ff.). Das heißt, dass es neben den 39 genannten Frauen noch weitere, nur versteckt beschriebene Frauen in den Lexika gibt.

In einem qualitativen Zugang ist die Frage interessant, welche acht Frauen im ersten Lexikon (Hergang, 1843-47) genannt werden. Den Genannten könnte man eine doppelte Pionierfunktion zuschreiben. Einmal mit Blick auf die Frage, wer es als Erste schafft und warum. Außerdem ist in vielen Vorwörtern zu lesen, dass man sich in der Arbeit auch auf vorangegangene Lexika stütze und sich also dort Anregungen holt. Deshalb könnte man annehmen, dass eine bereits einmal platzierte Frau höhere Chancen hat, wieder aufgenommen zu werden.

Dem scheint nicht so zu sein, denn vier der acht ersten Frauen werden in keinem der nachfolgenden Lexika mehr genannt (Anhalt, Hanke, Hoffmann, Schoppe); Elizabeth Hamilton wird noch einmal, Caroline Rudolphi noch zweimal und Albertine Adrienne Saussure de Necker und Pauline zur Lippe werden je noch drei Mal aufgeführt.Bei diesen acht Frauen handelt es sich um fünf Deutsche, eine Schweizerin, eine Britin und eine Polin, davon sind zwei, zum Zeitpunkt der Herausgabe des Lexikons, noch lebende Zeitgenossinnen. Die meisten sind evangelischen Glaubens. Beruflich sind vier Frauen als Erzieherin oder Lehrerin tätig, also aus heutiger Sicht in einem klassisch pädago- gischen Beruf, drei Frauen (Rudolphi, Schoppe, zur Lippe) haben Anstalten gegründet; die meisten allerdings, insgesamt sechs Frauen werden als pädagogische Schriftstellerinnen gewürdigt, hierin liegt oft die Rechtfertigung für ihre Aufnahme in das Lexikon „,unter die Reihen der Männer" (Hergang, 1847. S. 369.). Das heißt, die Tatsache, dass sie etwas über Erziehung geschrieben hat, scheint das stärkste Motiv gewesen zu sein, eine Frau in Hergangs Lexikon aufzunehmen. Das liest sich dann bei Caroline Christiane Louise Rudolphi (1753-1811) beispielsweise folgendermaßen: „Hier [in Heidelberg] fing die Dichterin ein Werk an, auf das sie lange zuvor mit Liebe gesonnen, und das ihr einen ehrenvollen Platz in der Reihe pädagogischer Schriftsteller gibt “ (ebd., S. 537.). Demnach ist es "ehrenvoll“ in diesen Kreis aufgenommen zu werden. Diese Ehre wird ebenso Elizabeth Hamilton (1756-1816) zuteil, die mit nur wenigen Zeilen gewürdigt wird, wobei drei ihrer Schriften im Besonderen aufgeführt sind.

Die anderen vier Schriftstellerinnen sind Henriette Wilhelmine Hanke, geb. Arndt (1785-1862) , die als ,[e]ine durch ihre Schriften um die Förderung weiblicher Geistesund Herzensbildung sehr verdiente Frau" (ebd., S. 13.) gerühmt wird; Clementine Hoffmann, geb. Tanska (1798-1845) gilt den Herausgebern "als eine um die Verbesserung des weiblichen Erziehungswesens in Polen hochverdiente Frau und ausgezeichnete Schriftstellerin" (ebd., S. 41.; Sperrung im Original); Alber- 
tine Adrienne Saussure de Necker (1766-1841) wird mit einem Buch über die früheste Kindheit bis drei Jahre und einem über die Erziehung von Mädchen vorgestellt; und Amalie Emma Schoppe (1791-1858) wird für ihre Jugendschriften gerühmt. Geschätzt wird bei fast allen (fünf von sechs), dass ihre Schriften relevant für die Praxis sind, sie also beispielsweise „in dem Garten der Jugendbildung nützlich gewirkt haben" (ebd., S. 619.) oder aber „wohltätig auf die sittliche Bildung" (ebd., S. 47.) wirkten. Auch die Sittlichkeit und Moralität der Frauen bzw. ihrer Schriften wird besonders unterstrichen, so bei Hanke: "Alle ihre Erzählungen und Romane zeichnen sich durch ... die reinste Moral und Religiosität auf das Rühmlichste aus, und sind daher allen jungen Mädchen und Frauen als wahre Lehrbücher zur Vervollkommnung ihrer sittlichen und geistigen Bildung $\mathrm{zu}$ empfehlen" (S. 14.).

Dieser deutlichen Empfehlung steht entgegen, dass Hanke in keinem anderen Lexikon mehr genannt wird. Genauso ergeht es Amalie Schoppe, bei der in Hergang noch eine ausführliche Werkszusammenstellung mit 30 Literaturangaben aufgelistet wird. Eine weitere interessante Beobachtung ist, dass zwei dieser sechs Frauen eigene Erziehungspensionate hatten, was zwar gewürdigt wird - auch mit lobenden Worten - aber nicht ausschlaggebend für das Verdienst ist, in Hergangs Pädagogische Real-
Encyclopädie aufgenommen zu werden. D. h. sie werden sowohl als Heldinnen der Praxis ${ }^{14}$ als auch als Denkerinnen beschrieben, da deren Werke und Reflexionen - und das wird als wichtig herausgestellt - sich eignen die Praxis zu verbessern.

Als Kontrast zu diesem ersten frauenbeschreibenden Lexikon werfen wir im Folgenden noch einen Blick auf die drei letzten Lexika des Untersuchungszeitraums. Sie stammen alle vom Herausgeber Wilhelm Hehlmann, dessen Wörterbuch der Pädagogik bis heute neu aufgelegt wird, inzwischen unter Herausgeberschaft von Winfried Böhm ${ }^{15}$. Das Wörterbuch wurde in erster Auflage 1931, in zweiter 1941 und dritter Auflage 1942 herausgegeben, damit lassen sich die Wissensbestände diachron vergleichen. Hier kommt interessantes zutage: In der ersten Auflage finden sich fünf Frauen, in der zweiten drei und der dritten zwei.

Gertrud Bäumer, Charlotte Bühler, Helene Lange, Ellen Key und Maria Montessori werden von Hehlmann als wichtigste Vertreterinnen für das Gebiet der Erziehungswissenschaft vorgestellt. Zumindest kann man sie nach Wilhelm Hehlmanns Vorwort so bezeichnen, da er dort angibt, ,,... ein handliches und ... erschwingliches Taschenbuch ... über die wichtigsten Gebiete des Bildungswesens und seiner Organisation, der Theorie der Bildung und Erziehung, ... u.a." herauszugeben.

\footnotetext{
${ }^{14}$ Vgl. für den Sprachgebrauch von Helden und Denkern Dolch 1931.

${ }^{15}$ Die aktuellste Ausgabe ist die 16. Auflage: Böhm, 2005.
} 
Pedagógiatörténeti Szemle • 1. évf. 4. sz. 40-61. o. • 2015

DOI:10.22309/PTSZEMLE.2015.4.3

Tabelle 2: Lemmata zu Frauen in den ersten drei Auflagen des Wörterbuchs von Wilhelm Hehlmann

\begin{tabular}{|l|l|l|l|}
\hline & Hehlmann 1931 & Hehlmann 1941 & Hehlmann 1942 \\
\hline Bäumer, Gertrud (1873-1954) & $\mathrm{x}$ & & \\
\hline Bühler, Charlotte (1893-1974) & $\mathrm{x}$ & & \\
\hline Lange, Helene (1848-1930) & $\mathrm{x}$ & $\mathrm{x}$ & $\mathrm{x}$ \\
\hline Key, Ellen (1849-1926) & $\mathrm{x}$ & $\mathrm{x}$ & \\
\hline Montessori, Maria (1870-1952) & $\mathrm{x}$ & $\mathrm{x}$ & $\mathrm{x}$ \\
\hline
\end{tabular}

Die Erscheinungsjahre der Auflagen bieten einen möglichen Ansatz, um zu erklären, warum ein Teil der Frauen nicht durchgängig aufgeführt wird: Gertrud Bäumer als linksliberale Politikerin und Charlotte Bühler als Jüdin waren keine nationalsozialistisch anerkannten Persönlichkeiten.

Und Hehlmann bekennt sich in seiner zweiten Auflage nicht nur zum Nationalsozialismus, sondern möchte mit dem Wörterbuch sogar einen Beitrag leisten, den Nationalsozialismus langfristig zu etablieren (vgl. Hehlmann, 1941. S. VIIf.). So erscheint in den Auflagen von 1941 und 1942 das Lemma ,Juden', das in der ersten Auflage noch nicht vorkam. In diesem Lemma beschreibt Hehlmann die angebliche jüdische "Zersetzung der erzieherischen Werte" (Hehlmann, 1942. S. 206.) und zählt jüdisch Wissenschaftler Innen auf. Dabei betont er, dass insbesondere in der Psychologie viele Juden tätig waren, und hier findet sich dann auch Charlotte Bühler ${ }^{16}$ als eigens genannte Kinderpsycholo- gin wieder, obwohl sie kein eigenes Lemma mehr besitzt.

Warum allerdings Ellen Key ${ }^{17}$ in der Auflage von 1942 verschwindet, bleibt noch etwas rätselhaft. Um zu prüfen, welche der fünf Frauen nach dem Zweiten Weltkrieg sozusagen rehabilitiert wurde, wurde die nächste Auflage des Hehlmann'schen Wörterbuchs von 1953 herangezogen. Es zeigt sich, dass alle fünf Frauen wieder mit eigenem Stichwort genannt werden.Demnach eignen sich die Wörterbücher von Hehlmann, um zu zeigen, wie die Geschichte der Erziehungswissenschaft in ihrer Darstellung an ein Regime angepasst wird. So wird das Bild der RepräsentantInnen der Disziplin verändert dargestellt, ebenso passte Hehlmann die Literaturangaben an und zitierte NSkonforme Autoren.

\section{FAZIT}

Zwar ist die Geschichte der Theorien und Begriffe der Pädago-

\footnotetext{
${ }^{16}$ Hier ohne Verweis auf ihren Mann Karl Bühler, der sonst immer einen eigenen Eintrag nach seiner Frau hat (vgl. Hehlmann 1931, 1953).

${ }^{17}$ Möglicherweise könnte ein Grund sein, dass sie nicht aus einem der mit Deutschland aktiv verbündeten Staaten stammte.
} 
gik/Erziehungswissenschaft gut erforscht, etwa in Form von Theorieund Begriffsgeschichten oder Klassikerkompendien. Auch die Disziplingeschichte ist insbesondere mit einem Fokus auf Strukturen und Institutionen bereits gut erhellt. Mit einem Fokus auf die erziehungswissenschaftlichen Wissensbestände in fachlexikalischen Formaten können die beiden Dimensionen von Theorien und Begriffen der Disziplin sowie der Disziplingeschichte aber noch ergänzt werden.

Notwendig, das zeigte der Versuch, die Lexika in ihren Intentionen und Positionierungen im wissenschaftstheoretischen Diskurs der 1970er Jahren zu verorten, ist es aber immer, die Heterogenität der Lexika zu beachten. Für die Ebene der Wissensbestände ergeben sich hieraus leitende Fragen, zum Beispiel: Werden einzelne Wissensbestände von bestimmten Gruppen oder zu bestimmten Zeiten unter anderen Stichworten subsumiert? Oder verschwinden mit Begriffen auch die ihnen zugeordneten Theorien und Ideen, wandern sie ab? Konkurrieren Begriffe um dieselben Wissensbestände? Werden die gesetzten und in der Analyse rekonstruierbaren Wissensbestände von je unterschiedlichen Herausgebern unterschiedlich formiert? Die Vielfalt der Programmatiken, die sich für das Jahrzehnt der 1970er Jahre zeigte, lässt sich nach einer ersten Durchsicht auch für andere Zeitabschnitte feststellen.

Am Beispiel der Frauen in den Lexika fällt etwas auf: In der moder- nen Debatte um Klassiker und Klassikerinnen wird häufig gefragt, wer denn nun zu dieser Gruppe gehört. Ohne eine Antwort auf diese Frage geben zu wollen, kann im Vergleich für die Frauen festgestellt werden, dass in heutigen KlassikerInnensammlungen (vgl. Tenorth, 2010 u.a.) z.B. Alice Salomon und Jane Addams aufgeführt werden, die in den Lexika nicht auftauchen. Diese Leerstellen lassen die Frage nach dem Warum aufkommen. Eine vorläufige, noch zu erhärtende Antwort auf diese Frage wäre: Die Klassikerzuschreibung erfolgt wohl erst in späteren Generationen - und wird nicht von Zeitgenossen geteilt. Eine intensivere Forschung hierzu und auch zu den männlichen Personen in Lexika mag einen Beitrag zur Klassiker-Debatte in der Erziehungswissenschaft bieten. Eine Sichtung und Auswertung von ausgewählten Personenartikeln zeigte bereits, dass diese wiederum zur Erläuterung des Theoriebestandes der Disziplin genutzt werden können, da dort nicht nur Biografisches, sondern auch theoretische und begriffliche Inhalte präsentiert und diskutiert werden.

Durch die serielle Analyse von fachlichen Nachschlagewerken über große Zeiträume und unterschiedliche wissenschaftstheoretische und programmatische Hintergründe hinweg, kann die Frage nach dem Stellenwert einzelner Wissensbestände etwa des Begriffs Erziehung oder der verhandelten Personen - in der sich zur Disziplin entwickelnden Erziehungswissenschaft, nach Kontinuitä- 
ten, Diskontinuitäten und Konjunkturen bearbeitet werden. Die Diskussion der Wissensbestände kann so als disziplinäres Phänomen analysiert werden.

\section{LITERATUR}

Baumgarten, J. C. F. (1828): Handwörterbuch für Volksschullehrer oder Belehrungen über Erziehung und Unterricht [...]. Zwei Theile. Quedlinburg, Leipzig.

Böhm, W. (2005): Wörterbuch der Pädagogik. 16., vollst. überarb. Auflage. Stuttgart.

Brachmann, J. (2008): Der pädagogische Diskurs der Sattelzeit. Eine Kommunikationsgeschichte. Bad Heilbrunn.

Bühler, P. (2012): „Vom Nutzen und Vorteil der Historien für das Leben." Vorreden und Einleitungen zu deutschen Geschichten der Pädagogik des 19. Jahrhunderts. Paedagogica Historica. International Journal of the History of Education, 48. 5. 676-691.

Bühler, P. (2014): Einführungen in die Pädagogik. Die Entwicklung einer Gattung. In: Fatke, R. und Oelkers, J. (Hrsg.): Das Selbstverständnis der Erziehungswissenschaft. Geschichte und Gegenwart. 60. Beiheft der Zeitschrift für Pädagogik. Weinheim u. a. 33-46.

Caspar, A. (1931): Lange, Helene. In: Schwartz, H. (Hrsg.) (1928-31): Pädagogisches Lexikon. Vierter Band. Rechtschreibung - Zwingli. Bielefeld, 1376-1379.

Deutsches Institut für wissenschaftliche Pädagogik (Münster) (DI${ }_{w P}$ ), Institut für Vergleichende Erziehungswissenschaft (Salzburg) (IVE) (1952): Geleitwort. In: Deutsches In- stitut für wissenschaftliche Pädagogik (Münster), Rombach, H. (Hrsg.): Lexikon der Pädagogik. Bd. 1: Systematischer Teil Abb-Fertigkeit. Freiburg im Breisgau, V-VI.

Deutsches Institut für wissenschaftliche Pädagogik (Münster) (DIwP), Speck, J. und Wehle, G. (1970): Handbuch pädagogischer Grundbegriffe. 2 Bde. München.

Deutsches Institut für wissenschaftliche Pädagogik (Münster), Rombach, Heinrich (1952-55/1964): Lexikon der Pädagogik. Freiburg im Breisgau.

Dolch, J. (1930): Gegenstände und Formen der Pädagogischen Geschichtsschreibung. Zeitschrift für Geschichte der Erziehung und des Unterrichts, 20. 1, 275-300.

(online: http:/ /nbn-resolving.de/urn/ resolver.pl?urn=urn:nbn:de:0111-

bbf-spo-6489033, letzter Zugriff 08.03.2012)

Gast, H., Leugers, A. und LeugersScherzberg, A. H. (2010): Optimierung historischer Forschung durch Datenbanken. Die exemplarische Datenbank „Missionsschulen 1887-1940". Bad Heilbrunn.

Groothoff, H.-H. (1964): Einleitung. In: Groothoff, H.-H. und Reimers, E. (Hrsg.): Das Fischer-Lexikon. 36. Pädagogik. Frankfurt am Main. 7-10.

Groothoff, H.-H. und Stallmann, M. (1961): Vorwort der Herausgeber. In: Groothoff, H.-H. und Stallmann, M. (Hrsg.): Pädagogisches Lexikon. Mit einem Anhang über die Geschichte der Pädagogik und über das Bildungswesen der Länder. Stuttgart, o. S. 
Groothoff, H.-H. und Stallmann, M. (1971): Vorwort. In: Groothoff, H.H. und Stallmann, M. (Hrsg.): Neues pädagogisches Lexikon. Stuttgart, Berlin, o. S.

Groothoff, H.-H. und Reimers, E. (1973): Das Fischer-Lexikon. 36. Pädagogik. Frankfurt am Main.

Groothoff, H.-H. und Stallmann, M. (1961): Pädagogisches Lexikon. Stuttgart.

Groothoff, H.-H. und Stallmann, M. (1971): Neues pädagogisches Lexikon. Stuttgart, Berlin.

Groothoff, H.-H. und Stallmann, M. (1964): Pädagogisches Lexikon. Stuttgart.

Großkopf, S. (2012): Industrialisierung der Pädagogik. Eine Diskursanalyse. Würzburg.

Hehlmann, W. (1931): Pädagogisches Wörterbuch. Leipzig.

Hehlmann, W. (1941): Pädagogisches Wörterbuch. Stuttgart.

Hehlmann, W. (1941): Vorwort. In: Hehlmann, W. (Hrsg.): Pädagogisches Wörterbuch. Stuttgart. VII-IX.

Hehlmann, W. (1942): Juden. In: Hehlmann, W. (Hrsg.): Pädagogisches Wörterbuch. Stuttgart. 206-207.

Hehlmann, W. (1942): Pädagogisches Wörterbuch. Stuttgart.

Hehlmann, W. (1953): Pädagogisches Wörterbuch. Stuttgart.

Hergang, K. G. (Red.) (1843-47): Pädagogische Real-Encyclopädie oder Encyclopädisches Wörterbuch des Erziehungs- und Unterrichtswesens und seiner Geschichte: für Lehrer an Volksschulen und andern Lehranstalten, für Eltern und Erzieher, für Geistliche, Schulvorsteher und andere Freunde der Päd- agogik und des Schulwesens bearbeitet von einem Vereine von Predigern und Lehrern. 2 Bde. Grimma.

Hergang, K. G. (Red.) (1847): Pädagogische Real-Encyclopädie oder Encyclopädisches Wörterbuch des Erziehungsund Unterrichtswesens und seiner Geschichte: für Lehrer an Volksschulen und andern Lehranstalten, für Eltern und Erzieher, für Geistliche, Schulvorsteher und andere Freunde der Pädagogik und des Schulwesens bearbeitet von einem Vereine von Predigern und Lehrern. Zweiter Band Haas - Ulrich Zwingli. Grimma.

Herzog, W. (2005): Pädagogik und Psychologie im Wörterbuch. Zur Normalität der Erziehungswissenschaft. Zeitschrift für Pädagogik 51. 673-693.

Hierdeis, H. (1978): Taschenbuch der Pädagogik. 2 Bde. Baltmannsweiler.

Horney, W., Ruppert, J. P., Schultze, W. und Scheuerl, H. (1970): Pädagogisches Lexikon. 2 Bde. Gütersloh.

Ipfling, H.-J. (1974): Grundbegriffe der pädagogischen Fachsprache. München.

Jacobmeyer, W. (2011): Das deutsche Schulgeschichtsbuch 1700-1945. Die erste Epoche seiner Gattungsgeschichte im Spiegel der Vorworte. 3 Bände. Berlin.

Keiner, E. (1999): Erziehungswissenschaft 1947-1990. Eine empirische und vergleichende Untersuchung zur kommunikativen Praxis einer Disziplin. Weinheim.

Keller, J. A. und Novak, F. (1979): Vorwort. In: Keller, J. A. und Novak, F. (Hrsg.): Kleines pädagogisches Wörterbuch. 55 Hauptartikel, 502 Definitionsartikel; ausführliches Verweisregister, aktuelle Literaturhinweise. Freiburg im 
Breisgau. 7-8.

Küster, C. D. (1774): Sittliches Erziehungs-Lexicon, oder Erfahrungen und geprüfte Anweisungen: wie Kinder von hohen und mittlern Stande, zu guten Gesinnungen und zu wohlanständigen Sitten können angeführet werden. Magdeburg.

Leimgruber, Y. (2006): In pädagogischer Mission. Die Pädagogin Rosette Niederer-Kasthofer (1779-1875) und ihr Wirken für ein "frauengerechtes" Leben in Familie und Gesellschaft. Bad Heilbrunn.

Lenzen, D. und Rost, F. (1999): Die Fachlexikographie der Pädagogik/Erziehungswissenschaft. In: Hoffmann, Kalverkämper und Wiegand, 2. Halbband. Berlin u.a.. 2013-2036.

Lenzen, D. und Mollenhauer, $\mathrm{K}$. (1982-86): Enzyklopädie Erziehungswissenschaft. Handbuch und Lexikon der Erziehung in 11 Bänden und einem Registerband. 12 Bde. Stuttgart.

Lenzen, D. und Rost, F. (1989): Pädagogische Grundbegriffe. 2 Bde. Reinbek bei Hamburg.

Münch, M. C. (1840-42): UniversalLexicon der Erziehungs- und UnterrichtsLehre für ältere und jüngere christliche Volksschullehrer. 3 Bde. Augsburg.

Münch, M. C. (1844-45): UniversalLexicon der Erziehungs- und UnterrichtsLehre für ältere und jüngere christliche Volksschullehrer, Schulkatecheten, Geistliche und Erzieher. Augsburg.

Münch, M. C. und Loé, H. T. (1858-60): Universal-Lexicon der Erziehungs- und Unterrichts-Lehre für ältere und jüngere christliche Volksschullehrer. Augsburg.

Rauch, E., und Anzinger, W.
(1973): Wörterbuch kritische Erziehung. Starnberg.

Rauch, E. und Anzinger, W. (1975): Wörterbuch kritische Erziehung. Starnberg.

Rein, W. (Hrsg.) (1895-99): Encyklopädisches Handbuch der Pädagogik. 7 $B d e$. Langensalza.

Rein, W. (Hrsg.) (1903-11): Encyklopädisches Handbuch der Pädagogik. 10 $B d e$. Langensalza.

Rolfus, H. und Pfister, A. (1863-66): Real-Encyclopädie des Erziehungs- und Unterrichtswesens nach katholischen Principien. Unter Mitwirkung von geistlichen Schulmännern für Geistliche, Volksschullehrer, Eltern und Erzieher. 4 Bde. Mainz.

Roloff, E. M. (Hrsg.) (1913-17): Lexikon der Pädagogik. 5 Bde. Freiburg im Breisgau.

Rombach, H. (1977): Vorwort. In: Willman-Institut München - Wien, Rombach, H. (Hrsg.): Wörterbuch der Pädagogik. Band 1: Abendschulen bis Genetische Methode. Freiburg im Breisgau, Basel, Wien. o. S.

Rost, F. (2008): Zur Lemmaund Inhaltsanalyse pädagogischer Nachschlagewerke. In: Ehrenspeck, Y./Haan, G. de/Thiel, F. (Hrsg.): Bildung: Angebot oder Zumutung? Wiesbaden, 141-158.

Roth, L. (1976): Handlexikon zur Erziehungswissenschaft. München.

Sander, K. H. P. F. (1883): Lexikon der Pädagogik: Handbuch für Volksschullehrer, enthaltend das Ganze des Unterrichts-und Erziehungswesens, Didaktik, Methodik. Leipzig.

Sander, K. H. P. F. (1889): Lexikon der Pädagogik: Handbuch für Lehrer 
und Erzieher, enthaltend das Ganze des Unterrichts- und Erziehungswesens in kurzer, alphabetisch geordneter Übersicht. Leipzig.

Schmid, K. A. (Hrsg.) (1859-78): Encyklopädie des gesammten Erziehungsund Unterrichtswesens bearbeitet von einer Anzahl Schulmänner und Gelehrten, unter Mitwirkung von Palmer, Wildermuth, Hauber u.a. 11 Bde. Gotha.

Schmid, K. A. (Hrsg.) (1876-87): Encyklopädie des gesammten Erziehungsund Unterrichtswesens bearbeitet von einer Anzahl Schulmänner und Gelehrten, unter Mitwirkung von Palmer, Wildermuth, Hauber u.a. 2., verb. Auflage. 10 Bde. Gotha.

Schneider, F. und Haase, K. (1964): Vorwort. In: Deutsches Institut für wissenschaftliche Pädagogik (Münster)/Rombach, H. (Hrsg.): Lexikon der Pädagogik. Bd. 5: Ergänzungsband. Freiburg im Breisgau, V.

Schwartz, H. (Hrsg.) (1928-31): Pädagogisches Lexikon. 4 Bde. Bielefeld.

Selbmann, R. (2006): "Zur Hebung des Natur-, Heimats- und Vaterlandsgefühls". Lesebuch-Vorworte zwischen Kaiserreich und Drittem Reich; eine Fallstudie. In: Korte, $\mathrm{H}$. (Hrsg.): Das Lesebuch 1800-1945. Ein Medium zwischen literarischer Kultur und pädagogischem Diskurs. Vorträge des 2. Siegener Symposiums zur literardidaktischen Forschung. Frankfurt am Main. 261-273.

Speck, J. und Wehle, G. (1970): Vowort der Herausgeber. In: Deutsches Institut für wissenschaftliche Pädagogik (DIwP), Speck, J. und Wehle, G. (Hrsg.): Handbuch pädagogischer Grundbegriffe. Band 1. München, V-VIII.
Speichert, H. (1975): Kritisches Lexikon der Erziehungswissenschaft und Bildungspolitik. Reinbek bei Hamburg.

Spieler, J. (Hrsg.) (1930-32): Lexikon der Pädagogik der Gegenwart. 2 Bde. Freiburg im Breisgau.

Stisser, A., Hild, A., Schindler, C. und Ell, B. (2013): Neue Forschungswerkzeuge in der Historischen Bildungsforschung. Die virtuelle Forschungsumgebung SMW-CorA für die kollaborative Analyse und Auswertung umfangreicher digitalisierter Quellen. In: Jahrbuch für Historische Bildungsforschung, 19. 305-325.

Stroß, A. M. und Thiel, F. (1998): Themenkonjunkturen und Disziplinentwicklung. Eine Untersuchung erziehungswissenschaftlicher Zeitschriftenbeiträge 1987-1994. In: Stroß, A. M., Thiel, F. und Appelsmeyer, $\mathrm{H}$. (Hrsg.): Erziehungswissenschaft, Nachbardisziplinen und Öffentlichkeit. Themenfelder und Themenrezeption der allgemeinen Pädagogik in den achtziger und neunziger Jahren. Weinheim. 9-32.

Tenorth, H.-E. (1986): Transformationen der Pädagogik. 25 Jahre Erziehungswissenschaft in der "Zeitschrift für Pädagogik". In: Fatke, R. (Hrsg.): 20. Beiheft der Zeitschrift für Pädagogik. Gesamtregister. Jahrgang 1-30 (1955-1984). Verzeichnis der Beiträge und Rezensionen mit einem Schlagwortregister sowie mit einer Chronik und einer Inhaltsanalyse. Weinheim.

Tenorth, H.-E. (2001): Zynismus oder das letzte Wort der Pädagogik. Zeitschrift für Pädagogik, 47. 4. 439-453.

Tenorth, H.-E. (2010, Hrsg.): Klassiker der Pädagogik. Band 1: Von Erasmus bis Helene Lange. München. 
Thadden-Trieglaff, R. von (1961): Geleitwort des Präsidenten des Deutschen Evangelischen Kirchentages. In: Groothoff, H.-H. und Stallmann, M. (Hrsg.): Pädagogisches Lexikon. Mit einem Anhang über die Geschichte der Pädagogik und über das Bildungswesen der Länder. Stuttgart. o. S.

Weber, E.,Domke, H. und Gehlert, S. (1974): Kleines sozialwissenschaftliches Wörterbuch für Pädagogen. Donauwörth.

Willman-Institut München, Wien (1970): Vorwort. In: WillmanInstitut München - Wien, Rombach, H. (Hrsg.): Lexikon der Pädagogik. Neue Ausgabe. Erster Band. ABC - Frankl. Freiburg im Breisgau. V-VI.

Willmann-Institut München, Wien,
Rombach, Heinrich (1977): Wörterbuch der Pädagogik. 3 Bde. Freiburg im Breisgau, Basel, Wien.

Willmann-Institut München, Rombach, Heinrich (1970-71): Lexikon der Pädagogik in vier Bänden. 4 Bde. Freiburg im Breisgau.

Wörle, J. G. C. (1835): Encyklopädisch-pädagogisches Lexikon oder vollständiges, alphabetisch geordnetes Hand- und Hilfsbuch der Pädagogik und Didaktik; zum Behuf des praktischen Lehrfachs, so wie zu Conferenz-Aufsätzen und Examina für Volks-Lehrer und Seminaristen, nach den besten Quellen und dem neuesten Standpunkt der Literatur. Heilbronn.

Wulf, C. (1974): Wörterbuch der Erziehung. München. 